\title{
Molecular signature of highly conductive metal-molecule-metal junctions
}

\author{
O. Tal, ${ }^{*}$ M. Kiguchi, ${ }^{\dagger}$ W. H. A. Thijssen, D. Djukic, C. Untiedt,,${ }^{\ddagger}$ R. H. M. Smit, and J. M. van Ruitenbeek \\ Kamerlingh Onnes Laboratory, Leiden University, P.O. Box 9504, 2300 RA Leiden, The Netherlands
}

(Received 10 October 2008; revised manuscript received 9 July 2009; published 24 August 2009)

\begin{abstract}
The simplicity of single-molecule junctions based on direct bonding of a small molecule between two metallic electrodes makes them an ideal system for the study of fundamental questions related to molecular electronics. Here we study the conductance properties of six different types of molecules by suspending individual molecules between Pt electrodes. All the molecular junctions show a typical conductance of about $1 G_{0}$ which is ascribed to the dominant role of the Pt contacts. However, despite the metalliclike conductivity, the individual molecular signature is well expressed by the effect of molecular vibrations in the inelastic contribution to the conductance.
\end{abstract}

DOI: 10.1103/PhysRevB.80.085427

PACS number(s): 73.63.Rt, 72.10.Di, 73.40.-c

\section{INTRODUCTION}

When forming a conductive molecular bridge between two metallic electrodes there is a tradeoff between the preservation of the molecular electronic structure and the realization of highly conductive molecular junctions. The first quality can be achieved by molecules that are connected to the electrodes via anchoring side groups ${ }^{1}$ (e.g., Au/benzene dithiol $^{2}$ ) which act as potential barriers that decouple to a large extent the molecule from both contacts. Such junctions have a conductance in the tunneling regime (about $10^{-5}-10^{-2} G_{0}$, where $G_{0}=2 e^{2} / h$ is the conductance quantum). ${ }^{3}$ The second quality is accomplished by simple molecules connected directly to the electrodes (e.g., a Pt/ $\mathrm{H}_{2}$ junction ${ }^{4}$ ) which results in a strong molecule-electrode coupling. Such junctions have a conductance in the (quasiballistic) contact regime (about $\left.0.1-1 G_{0}\right)^{4-7}$ which is comparable to the conductance of metallic atomic junctions. ${ }^{8}$

The use of anchoring side groups enables a better a priori control over the transport properties of the molecular junction by chemical synthesis since the structure and properties of the isolated molecule are preserved to a large extent, and the orientation of the molecule with respect to the leads is determined mainly by the position of the anchoring groups on the molecule. In the case of small molecules that react directly with the electrodes, the whole molecule serves as an "anchoring group," thus it is prone to structural and electronic modifications and its orientation cannot be easily predicted. In some extreme cases new structures can be formed by molecular decomposition as in the case of metal atom chains decorated with oxygen atoms. ${ }^{9}$

The direct binding of simple molecules to the electrodes offers a valuable opportunity to explore some of the central questions related to electron transport through molecular junctions. ${ }^{6,710}$ The high conductivity and the relatively simple electronic and atomic structure of such junctions permit adapting experimental techniques that were originally developed for atomic point contacts (e.g., measurements of shot noise, ${ }^{11,12}$ conductance fluctuations, ${ }^{4,13}$ and subgap structure in superconducting contacts ${ }^{14,15}$ ) and explore the effect of different manipulations such as junction stretching and isotope substitution on inelastic spectroscopy. ${ }^{10,16,17}$ This variety of experimental tools extends the number of observed properties available for research. Moreover simple molecular junctions can be described to higher accuracy by theoretical calculations due to the limited size of these physical systems. The simplicity of the electronic structure may help to validate different approximations ${ }^{18-21}$ that simplify the calculations and provide intuitive models. ${ }^{19,22,23}$ Consequently the comparison between theory and experiment is more straightforward.

In view of the role that they may play as reference and model systems, we present here a comparative study of simple-molecule junctions based on hydrogen $\left(\mathrm{H}_{2}\right)$, water $\left(\mathrm{H}_{2} \mathrm{O}\right)$, carbon monoxide $(\mathrm{CO})$, carbon dioxide $\left(\mathrm{CO}_{2}\right)$, ethylene $\left(\mathrm{C}_{2} \mathrm{H}_{2}\right)$, and benzene $\left(\mathrm{C}_{6} \mathrm{H}_{6}\right)$ connected to platinum $(\mathrm{Pt})$ electrodes. We measured the typical conductance, conductance evolution during introduction of the target molecule, response to current-induced heating, inelastic spectroscopy, and its dependence on junction stretching. Throughout the paper we focus on the manifestation of the molecular characteristic properties in the conductance.

\section{EXPERIMENTAL TECHNIQUE}

The molecular junctions were formed using the mechanically controllable break junction (MCBJ) technique. ${ }^{24}$ Starting with a macroscopic Pt wire (polycrystalline, $0.1 \mathrm{~mm}$ diameter, $99.99 \%$ purity), a small notch was cut at the middle of the wire in order to fix a breaking point. The wire was glued on top of a bending substrate which is mounted in a three-point bending configuration inside a vacuum chamber that is pumped to a pressure below $1 \times 10^{-5}$ mbar. After cooling to about $4.5 \mathrm{~K}$, when a cryogenic vacuum is attained, the wire is broken at the notch by bending of the substrate onto which it has been fixed. The clean, freshly exposed metallic apexes are then brought back into contact by slightly relaxing the bending. With the use of a piezoelectric element the displacement of the two electrodes can be finely adjusted to form a stable contact of atomic size. Due to the bendable sample, the contact displacement is about $10^{3}$ times smaller than the piezodisplacement allowing subangstrom control over the contact separation. ${ }^{24,25}$ The low-temperature MCBJ provides: (a) clean metal contacts produced under cryogenic vacuum, (b) high mechanical stability of the atomic/ molecular junction, (c) precise control over the distance be- 
tween the electrodes, and (d) fast formation and break of atomic/molecular junctions that allows statistical treatment of measurements done over many different junctions in a short time.

Two-probe conductance $(I / V)$ measurements versus interelectrode separation (conductance traces) are done while repeatedly breaking the contact using a piezoelectric element (junction pulling speed: $\sim 400 \mathrm{~nm} / \mathrm{s}$ ). A bias voltage is given by a PC DAQ card (e.g., NI-PCI-6251, 1.25 Msample/s or NI-PCI-6030E, $100 \mathrm{Ksample/s)} \mathrm{and} \mathrm{the} \mathrm{cur-}$ rent signal is amplified by a current to voltage amplifier (SR570) and sent to the PC DAQ card. Differential conductance $(d I / d V)$ measurements were performed on the molecular junction using a lock-in amplifier (SR830). For this propose the bias voltage was modulated with a fixed modulation amplitude of $1 \mathrm{mV}$ and a frequency of $7 \mathrm{kHz}$, while sweeping the dc bias voltage using the PC DAQ card in the range of +100 to $-100 \mathrm{mV}$ and back. The output ac signal was amplified by the mentioned current-to-voltage amplifier before being introduced to the lock-in amplifier input. The ac signal at the modulation frequency was then collected by the PC DAQ card.

\section{INTRODUCTION OF MOLECULES}

The introduction of the molecules was done while keeping the metallic electrodes at a cryogenic temperature. The molecules were prepared in a different chamber at room temperature. As molecular sources in the liquid phase we used deionized ${ }^{26} \mathrm{H}_{2} \mathrm{O}$ and $\mathrm{C}_{6} \mathrm{H}_{6}(99.9 \%)$ placed in a quartz tube. These compounds were degassed by cycles of freezing, pumping, and thawing. Compounds in the gas phase $\left[\mathrm{H}_{2}\right.$ (99.999\%), $\mathrm{D}_{2} \quad$ (99.999\%; $99.7 \%$ isotope enriched), $\mathrm{CO}(99.995 \%), \mathrm{CO}_{2}(99.995 \%)$, and $\left.\mathrm{C}_{2} \mathrm{H}_{2}(99.5 \%)\right]$ were introduced from gas cylinders to a dozing chamber that was flushed with the gas and pumped several times to minimize contaminations. In order to admit the molecules to the Pt junction at cryogenic temperatures we have used a molecular dozer (presented schematically in Fig. 1) containing a stainless steel capillary ${ }^{27}$ located inside the insert tube under cryogenic vacuum with weak thermal coupling to room temperature and strong thermal coupling to the liquid-He bath. The dozer can be heated by a heater wire wrapped around its outer side (or by a thermocoax heater wire running all along its interior in other versions). A short removable copper tube with a side nozzle (see Fig. 1 inset) is connected to the end of the main capillary. This part can be removed when the sample is replaced and it is designed to emit a molecular jet in a wide angle toward the junction. The molecular dozer and the gas dozing chamber were baked out before the introduction of molecules. The dozer temperature is monitored at four different locations along the capillary by Pt100 resistors ${ }^{28}$ and a single $\mathrm{RuO}_{2}$ resistor ${ }^{29}$ at the cold lower end. This design provides an efficient and controllable heating of the capillary up to $360 \mathrm{~K}$ and fast cooling below $20 \mathrm{~K}$. During heating the sample temperature rises by less than $4 \mathrm{~K}$ for a few minutes, as is typical when introducing water. The introduction of the other molecules involves less heating and the introduction of $\mathrm{H}_{2}$ or $\mathrm{D}_{2}$ can be done while the capillary maintains its base temperature.

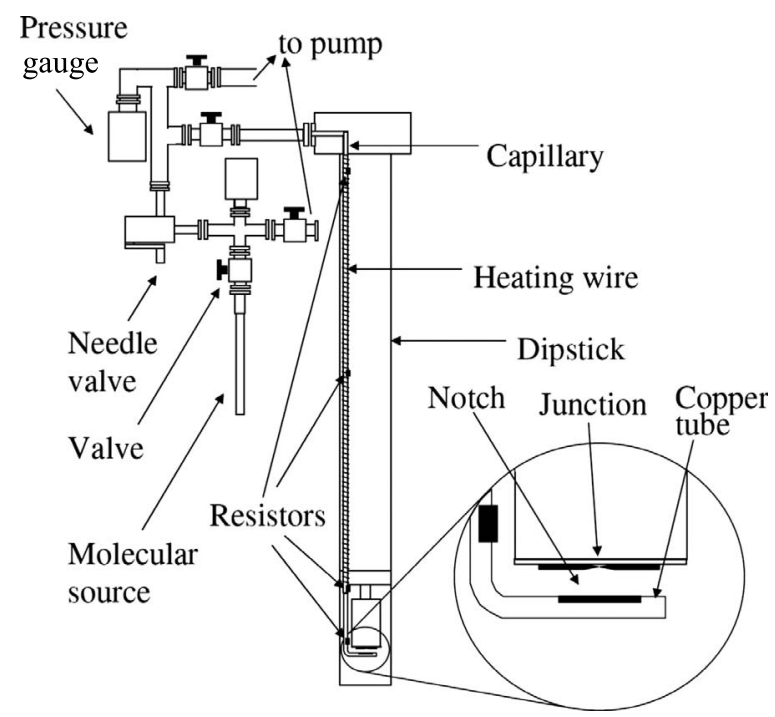

FIG. 1. Schematic representation of the molecule dozer and its location within the cryogenic insert.

While the Pt junction was broken and formed repeatedly, molecules were introduced to the junction through the molecular dozer. First, the target molecules were introduced to the cold capillary via a needle valve at the top of the capillary (illustrated in Fig. 1). Then the temperature of the capillary was gradually increased until a change in the conductance was detected (see the following section). The heating power to the capillary was kept constant for $1 \mathrm{~min}$. Then the heating was stopped and the capillary nozzle was allowed to cool down via the contact to the liquid-He bath. A rough estimation of the molecular deposition rate, assuming an equilibrium between the frozen target molecules on the capillary surface and the molecules that were released by heating, would be 0.05 molecules per minute per $\mathrm{nm}^{2}$ leading to a submonolayer coverage (on average). Note that the mechanical deformation and the released heat in the process of junction breaking and formation can assist the introduction of molecule to the junction. In the case of $\mathrm{H}_{2}$ around $10 \mu$ mol were introduced to a cold capillary but the dose arriving at the atomic contact cannot be determined very precisely due to finite vapor pressure at the base temperature of $4.5 \mathrm{~K}$.

\section{CONDUCTANCE HISTOGRAMS}

Before the introduction of molecules the formation of a clean $\mathrm{Pt}$ contact is verified by conductance histograms made from at least 1000 conductance traces taken during repeated contact stretching as presented in Fig. 2(a) (black curve). The single peak around $1.5 G_{0}$ provides a fingerprint of a clean $\mathrm{Pt}$ contact. ${ }^{30}$ The introduction of the target molecule is signaled by the suppression of the typical peak for Pt and the appearance of a new distribution of peaks (Fig. 2, filled curves). ${ }^{4,31}$ The peak locations for each of the molecular junction is given in Table I. Among the six junctions $\mathrm{Pt} / \mathrm{H}_{2} \mathrm{O}$ and $\mathrm{Pt} / \mathrm{CO}$ have a somewhat more distinct character: the conductance histogram for $\mathrm{Pt} / \mathrm{H}_{2} \mathrm{O}$ junctions is characterized by several 

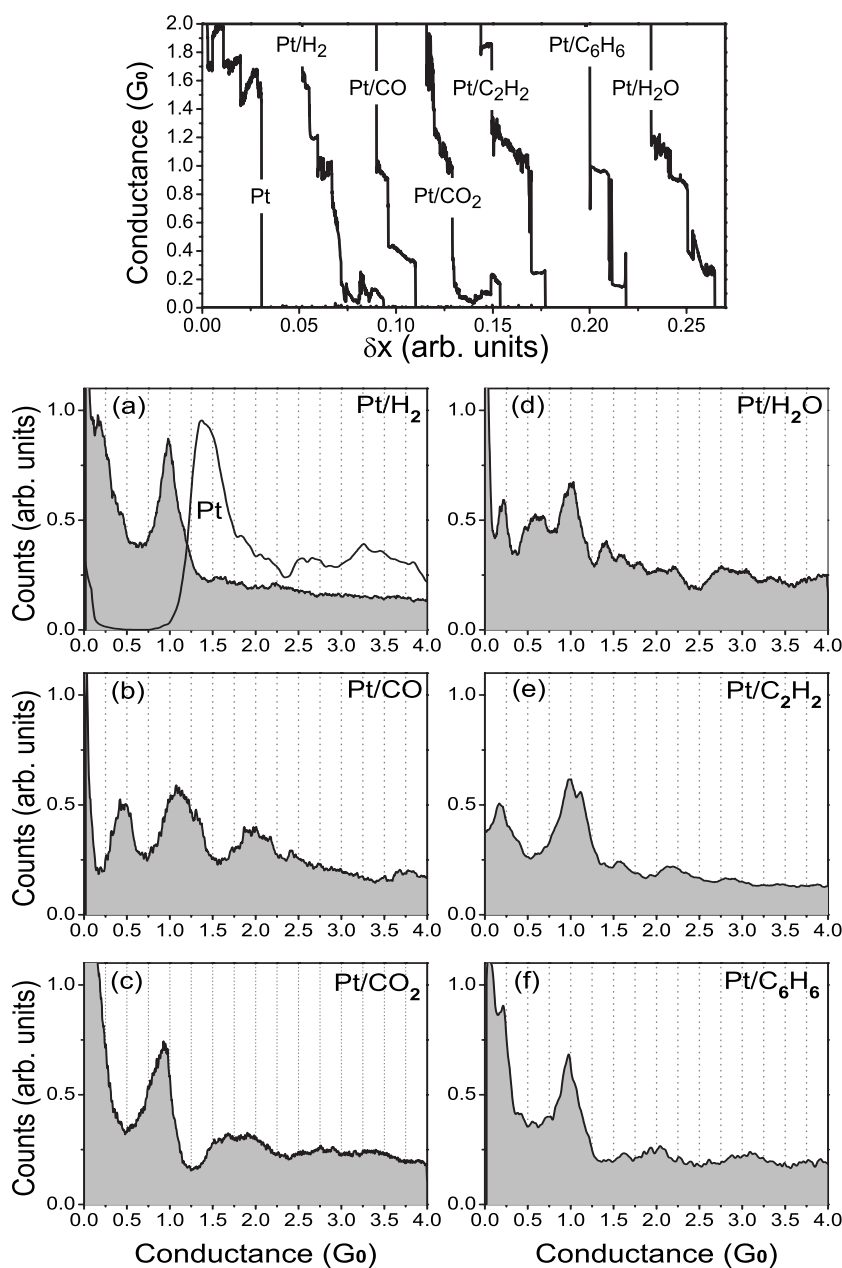

FIG. 2. Conductance versus displacement traces of Pt junctions before (left curve) and after the introduction of each molecule type. Each trace is composed from 5000 data points and located arbitrarily along the displacement axis (top). Conductance histograms for Pt junctions (normalized to the area under the curves) before (a, black curve) and after the introduction of (a) $\mathrm{H}_{2}$, (b) $\mathrm{CO}$, (c) $\mathrm{CO}_{2}$, (d) $\mathrm{H}_{2} \mathrm{O}$, (e) $\mathrm{C}_{2} \mathrm{H}_{2}$, and (f) $\mathrm{C}_{6} \mathrm{H}_{6}$ (shaded curves). Each conductance histogram is constructed from more than 1000 conductance traces (bottom).

relatively low peaks, which implies a variety of stable junction configurations. In some cases instead of the $1.0 G_{0}$ peak, two peaks at 0.90 and $1.10 G_{0}$ can be detected. $\mathrm{Pt} / \mathrm{CO}$ has a unique peak at around $0.5 G_{0}$ that appears in about $45 \%$ of the cases. Interestingly, calculations ${ }^{32,33}$ cannot reproduce the $1 G_{0}$ peak in the conductance histograms of $\mathrm{Pt} / \mathrm{CO}$ while the
$0.5 G_{0}$ is successfully obtained and is attributed to an asymmetric configuration where each atom $(\mathrm{C}$ and $\mathrm{O})$ is attached to a different electrode.

A peak around $0.2 G_{0}$ appears in some of the histogram measurements for $\mathrm{Pt} / \mathrm{H}_{2}, \mathrm{Pt} / \mathrm{H}_{2} \mathrm{O}, \mathrm{Pt} / \mathrm{C}_{2} \mathrm{H}_{2}$, and $\mathrm{Pt} / \mathrm{C}_{6} \mathrm{H}_{6}$. For $\mathrm{Pt} / \mathrm{H}_{2}$ it is attributed to molecular junctions that involve molecules connected to a chain of $\mathrm{Pt}$ atoms, ${ }^{34}$ while for $\mathrm{Pt} / \mathrm{C}_{6} \mathrm{H}_{6}$ it is was argued to be associated with the conductance of the stretched molecular junction. ${ }^{7}$ The origin of the $0.2 G_{0}$ conductance peak in the histograms of $\mathrm{Pt} / \mathrm{H}_{2} \mathrm{O}$ and $\mathrm{Pt} / \mathrm{C}_{2} \mathrm{H}_{2}$ has not been studied.

All the conductance histograms in Fig. 2 reveal a peak at around $1 G_{0}$ while its fine location and shape slightly varies between different molecular junctions. The appearance of this common peak is rather surprising specifically for $\mathrm{Pt} / \mathrm{H}_{2}$ and $\mathrm{Pt} / \mathrm{H}_{2} \mathrm{O}$ for which it has been demonstrated that the conductance at the $1 G_{0}$ peak in the histogram is carried dominantly by a single conductance channel ${ }^{4,6}$ According to the Landauer equation $G=\sum_{i} G_{0} T_{i}$, where $T_{i}$ is the $i$ th transmission probability for an electron to cross the junction. For a single conductance channel, $G=G_{0} T$, thus the channel is fully open $(T=1)$ at $G=1 G_{0}$. In a simple picture of a molecule with discrete electronic levels located between two metal electrodes, the Fermi level of the source and drain electrodes need to be aligned with the center of a molecular level to have the maximum transmission probability ("fullresonance" case). In an alternative picture, electron-electron correlations can lead to a resonance at the Fermi level under specific conditions. ${ }^{35}$

Bearing these pictures in mind, one could argue that it is remarkable to have the special conditions for such a perfect resonance for different single-channel molecular junctions. In the case of molecular junctions with several dominant channels, having the same total conductance of $1 G_{0}$ for several different junctions is surprising as well when considering that each junction has its own individual channel composition. However, as described by Ferrer et al. ${ }^{33}$ a strong hybridization of the molecular levels with the $d$ band of the metallic electrodes leads to a transmission probability of about 1 for a wide energy range around the Fermi level even when the Fermi energy is not aligned with a molecular level. Thus, the dominant role of the metallic electrode leads to a common peak around $1 G_{0}$ for many molecules, where the individual signature of the molecules is limited to the fine structure of the conductance histogram.

Note that an alternative scenario of a metal-to-metal contact decorated with molecules, or accompanied with a secondary conductance channel via a metal-molecule-metal bridge is unlikely. The main conductance peak, which is

TABLE I. Conductance (in units of $G_{0}$ and $\pm 0.05 G_{0}$ uncertainty) of the main peaks in the conductance histograms of the studied molecular junctions.

\begin{tabular}{cccccc}
\hline \hline $\mathrm{H}_{2}$ & $\mathrm{CO}$ & $\mathrm{CO}_{2}$ & $\mathrm{H}_{2} \mathrm{O}$ & $\mathrm{C}_{2} \mathrm{H}_{2}$ & $\mathrm{C}_{6} \mathrm{H}_{6}$ \\
\hline 0.20 & 0.46 & 0.94 & 0.20 & 0.22 & 0.21 \\
1.00 & 1.10 & & 0.60 & 1.04 & 0.98 \\
& & & 1.00 & & \\
\hline
\end{tabular}


found around $1 G_{0}$ for the molecular junctions, lies much lower than the conductance of a Pt-Pt contact (around $1.5 G_{0}$ ). Tuning the distance between Pt electrodes to achieve $1 G_{0}$ conductance is almost impossible-in this case the conductance either jumps above $1.4 G_{0}$ or to tunneling conductance below $0.1 G_{0} \cdot{ }^{30}$ In general terms, the size of a measured inelastic contribution to the current due to molecular vibrations is $1 \%-10 \%$. When the current path through the molecule takes only a small fraction of the total current ( $<10 \%$ ), as expected for the mentioned scenario, an inelastic contribution of $1 \%-10 \%$ to the total current due to that channel would become unphysically large which makes this scenario less likely.

\section{THE EVOLUTION OF CONDUCTANCE HISTOGRAMS: FROM METALLIC CONTACT TO MOLECULAR JUNCTION}

The conductance histograms in Fig. 2 were taken at the end of the process of introducing the target molecules. When the molecules are first introduced to the metallic junction the conductance histogram is observed to evolve through a series of steps that were found to be similar for $\mathrm{H}_{2} \mathrm{O}, \mathrm{CO}, \mathrm{CO}_{2}$, and $\mathrm{C}_{6} \mathrm{H}_{6}$. The introduction of $\mathrm{C}_{2} \mathrm{H}_{2}$ was not studied in this aspect and the evolution of conductance histograms for $\mathrm{Pt} / \mathrm{H}_{2}$ junctions is too fast for collecting reliable statistical data related to the junction evolution. Here we focus on the evolution of sequential conductance histograms while $\mathrm{H}_{2} \mathrm{O}$ molecules are admitted to a Pt junction as presented in Fig. 3. A conductance histogram of a virgin Pt junction is first taken [see Fig. 3(a)]. Then the target molecules are introduced to the cold capillary followed by gradual heating of the capillary. When the temperature at the outer side of the capillary near the sample reaches $120 \mathrm{~K}$ (the thermocoax temperature could be higher), the main peak in the histogram shifts to lower conductance [in this case from 1.5 to $1.4 G_{0}$, as seen in Fig. 3(b)] while its amplitude is suppressed [Figs. 3(b) and $3(\mathrm{c})$ ]. Next, contributions around $1.8 G_{0}$ become more pronounced as demonstrated in Fig. 3(d). Finally the feature around $1.8 G_{0}$ is suppressed and a conductance histogram typical for the final molecular junction has evolved [Figs. $3(\mathrm{e})$ and $3(\mathrm{f})]$.

The shift of the main peak in Fig. 3(a) to lower conductance can stem from different origins: (1) stimulated formation of $\mathrm{Pt}$ chains by the presence of molecules around the metallic contact can lead to lower conductance around $1.2-1.4 G_{0} \cdot{ }^{36,37}$ Indeed a shift of the Pt peak to $1.2 G_{0}$ is sometimes observed; (2) a lower conductance can arise from a weakening of the Pt-Pt bonds at the Pt junction by the adsorption of molecules near the junction (due to electrostatic effects, charge transfer, or other perturbations). These are two mutually excluding possibilities since the first scenario involves strengthening of the Pt-Pt bonds leading to chain formation when the contacts are pulled apart, while the second scenario is based on bond weakening.

When the shifted peak is suppressed, the conductance around $1.8 G_{0}$ becomes more pronounced. This value agrees with the conductance through a single bond between two Pt electrodes ${ }^{36,38}$ or more specifically, between two pyramidal
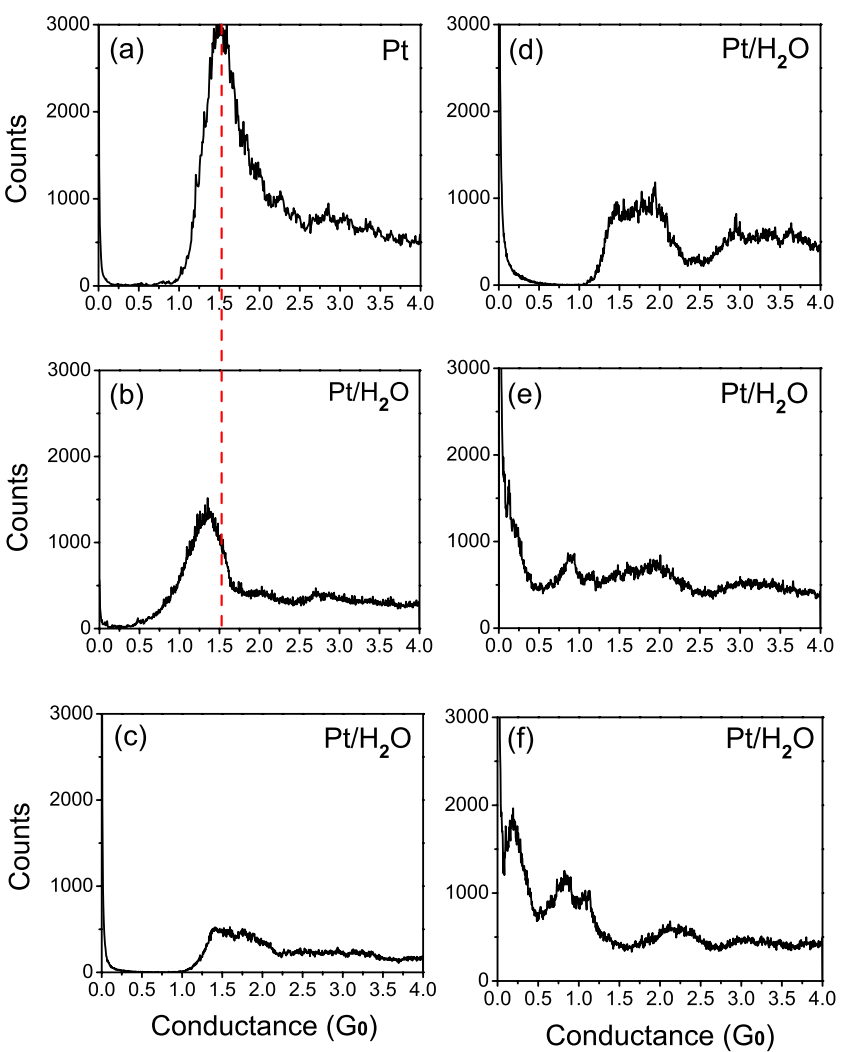

FIG. 3. (Color online) A series of sequential conductance histograms (i.e., each consequential histogram is restarted and does not contain data from the former one) for a virgin Pt junction (a) and a Pt junction after the introduction of $\mathrm{H}_{2} \mathrm{O}$ [(b)-(f)]. Each conductance histogram is constructed from 1000 conductance traces and takes about $6 \mathrm{~min}$

Pt electrodes. ${ }^{37}$ The number of counts below $1 G_{0}$ remains low which indicates that there are no molecular bridges being formed. This evolution step can be interpreted as a suppression of chain formation (which would give the $1.4-1.5 G_{0}$ peak) by the presence of molecules (i.e., weakening of the Pt-Pt bond). Thus the scenario of adsorbateinduced weakening of the Pt-Pt bonds at the junction can explain both the shift of the $1.5 G_{0}$ peak to lower conductance values and the increased probability for conductance at $1.8 G_{0}$ at the expense of the former peak. The sequence of events is in agreement with this scenario as well since moderate weakening of the Pt-Pt bond is expected to reduce conductivity while further weakening will lead eventually to bond breaking and suppression of chain formation.

\section{THE EFFECT OF CURRENT-INDUCED HEATING ON CONDUCTANCE HISTOGRAMS}

As demonstrated in Fig. 3 conductance histograms form an efficient tool in probing the presence of conducting molecules in the junction (and probably even the presence of molecules in the vicinity of the metallic junction). However, for most of the cases examined discrimination between the different molecules just from the conductance histogram is not trivial, as can be seen in Fig. 2. Thus, additional tools are 


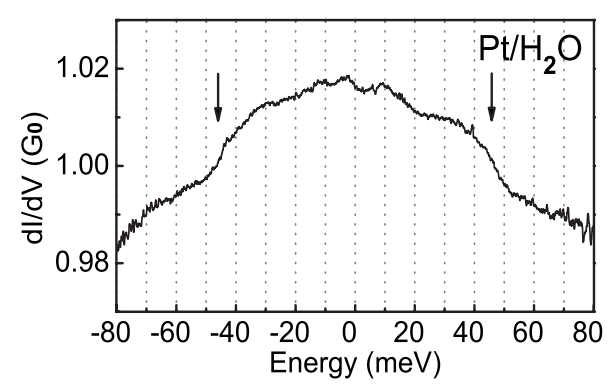

FIG. 4. Differential conductance $(d I / d V)$ as a function of applied voltage across a $\mathrm{Pt} / \mathrm{H}_{2} \mathrm{O}$ junction. The steps marked by the arrows are due to inelastic scattering by vibration modes in the molecule.

required in order to distinguish between them.

The evolution of the conductance histograms following application of high enough bias voltage (typically $1 \mathrm{~V}$ for 1 min) across the junction varies for different molecular junctions. For $\mathrm{Pt} / \mathrm{H}_{2}$ junction a typical histogram for clean $\mathrm{Pt}$ is recovered by application of such a high voltage. However, upon reducing the voltage below $300 \mathrm{mV}$ the typical conductance histogram of $\mathrm{Pt} / \mathrm{H}_{2}$ reappears. This behavior can be understood as the response to current-induced heating at the junction. ${ }^{39-41}$ As a consequence of local heating of the junction the hydrogen molecules diffuse away from the contact. However due to the finite diffusion rate of the light hydrogen at $4.5 \mathrm{~K} \mathrm{a} \mathrm{Pt} / \mathrm{H}_{2}$ junction is recovered once the heating has stopped. For $\mathrm{CO}, \mathrm{CO}_{2}$, and $\mathrm{C}_{6} \mathrm{H}_{6}$ the application of a high bias results in the recovery of a clean Pt histogram that remains stable even after reduction in the applied voltage. We ascribe the latter observation to the negligible diffusion rates for these molecules at $4.5 \mathrm{~K}$. Finally, high voltage usually does not affect the conductance histogram of $\mathrm{Pt} / \mathrm{H}_{2} \mathrm{O}$ junctions implying a relatively stable binding of the molecule to the electrodes. In some rare cases a Pt histogram can be obtained provided that the high voltage was applied a few seconds after the first indication for $\mathrm{H}_{2} \mathrm{O}$ in the junction. The difference in response following the application of a high bias voltage provides a quick method for obtaining an indication on the nature of the molecular junction.

\section{INELASTIC SPECTROSCOPY}

For inelastic spectroscopy the differential conductance $(d I / d V)$ is measured as a function of the applied voltage across a molecular junction. Figure 4 provides an example of a measured spectrum. When the voltage difference between the metal electrodes reaches a threshold given by a vibrationmode energy $(\hbar \omega)$ the electrons have enough energy to excite the molecular vibration mode. ${ }^{10,17}$ At this voltage $(\mathrm{eV}$ $=\hbar \omega)$ a step in the differential conductance appears due to the change in the transmission probability for electrons that interact with the molecular vibration ${ }^{6}$ (e.g., at $46 \mathrm{meV}$ in Fig. 4). The interpretation of the step in the $d I / d V$ signal in terms of changes induced by a molecular vibration mode has been confirmed by measurements of isotopes ${ }^{7,10} \mathrm{D}_{2}, \mathrm{HD}$, and ${ }^{13} \mathrm{C}_{6} \mathrm{H}_{6}$.

For each type of molecular junction we have collected many $d I / d V$ spectra where each measurement is performed
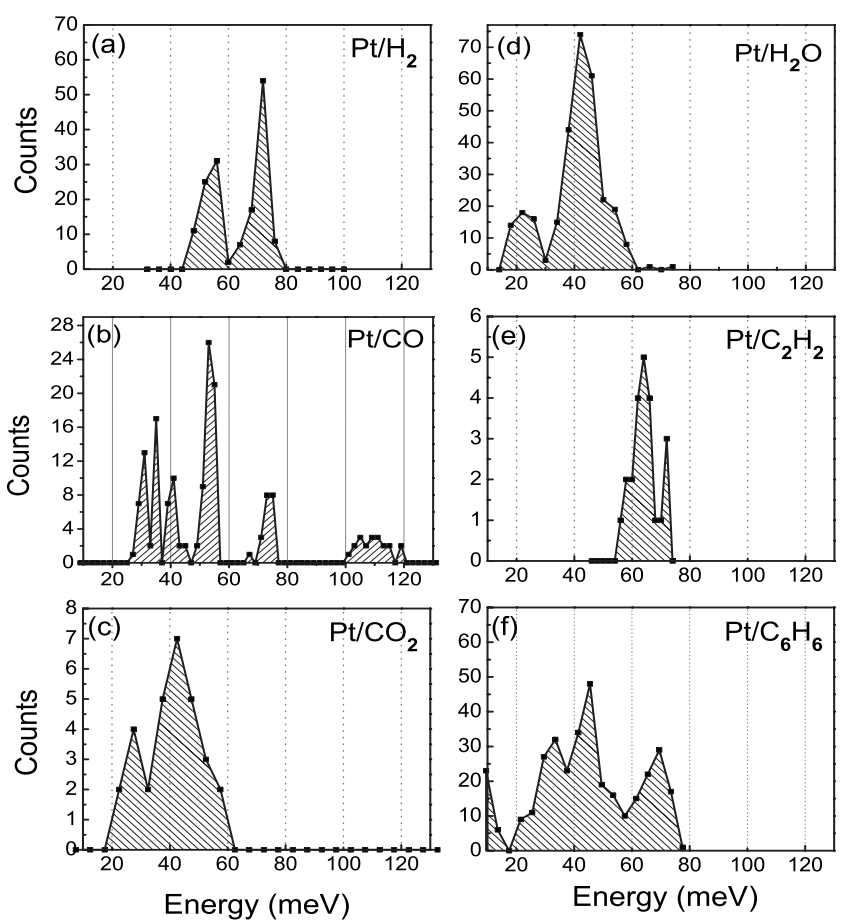

FIG. 5. Distribution of vibration-mode energies observed for (a) $\mathrm{H}_{2}$, (b) $\mathrm{CO}$, (c) $\mathrm{CO}_{2}$, (d) $\mathrm{H}_{2} \mathrm{O}$, (e) $\mathrm{C}_{2} \mathrm{H}_{2}$, and (f) $\mathrm{C}_{6} \mathrm{H}_{6}$ between $\mathrm{Pt}$ electrodes. The data was collected for junctions with zero-bias conductance below $1.1 G_{0}$ (for $\mathrm{C}_{6} \mathrm{H}_{6}$ this range is wider than appears in a previous report (Ref. 7) where the data was collected for zero-bias conductance below $0.4 G_{0}$ ). Note that for $\mathrm{Pt} / \mathrm{CO}$ and $\mathrm{Pt} / \mathrm{C}_{2} \mathrm{H}_{2}$ junctions only a limited set of data was collected.

on a newly formed junction. Figure 5 presents the number of times that a vibration mode with certain energy was detected for each molecular junction. The distribution of the typical vibration-mode energies reveals an individual signature for a specific molecular junction. The following two cases demonstrate the implications of such a chemical recognition.

On a few occasions, after several days of measurements taken on $\mathrm{Pt} / \mathrm{C}_{6} \mathrm{H}_{6}$ and also $\mathrm{Pt} / \mathrm{CO}$ junctions, unexpected features in the conductance histograms suggested a possible presence of $\mathrm{H}_{2} \mathrm{O}$ in the $\mathrm{Pt}$ junctions. The different response to current-induced heating experiments (see Sec. VI) supported this interpretation. However, the different vibrationenergy distributions for these junctions gave us much stronger indication for the presence of $\mathrm{H}_{2} \mathrm{O}$ as a contamination in the studied junctions. Thus the application of vibration-mode distribution as a chemical fingerprint of the junction allows us to deduce that substitution of the target molecules by contaminating molecules with higher affinity can take place even at low temperatures.

Chemical recognition is also important in cases where the conductance histogram of the target molecule is similar to that for junction based on a derivative of the target molecule as in the case of $\mathrm{Pt} / \mathrm{CO}$ and $\mathrm{Pt} / \mathrm{CO}_{2}$ junctions. The additional peak around $0.5 G_{0}$ for the $\mathrm{Pt} / \mathrm{CO}$ junction does not appear in all cases, leading to similarity between the conductance histograms for the two junctions. However, the large difference between the vibration-energy distributions for these junctions allows us to identify the presence of each molecule in the junction. 


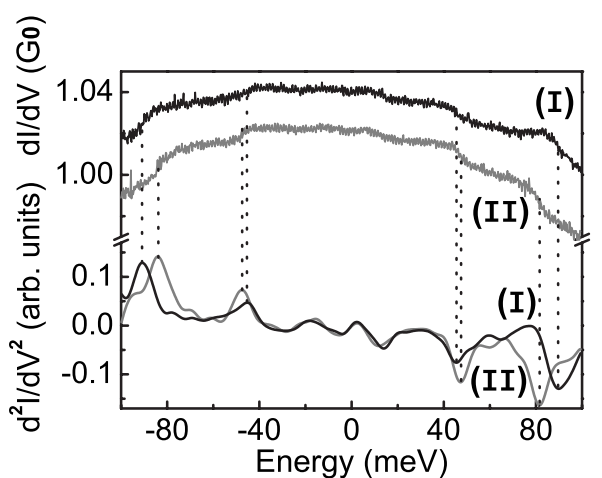

FIG. 6. $d I / d V$ (upper two curves) and corresponding numerical derivatives $d^{2} I / d V^{2}$ (lower two curves) as a function of the energy. Both measurements (I) and (II) are obtained on the same junction, except that the junction is stretched before the measurement (II).

\section{STRETCHING DEPENDENCE IN INELASTIC SPECTROSCOPY}

The MCBJ technique provides subatomic control on the distance between the electrodes while the mechanical stability of the junction is maintained. When using MCBJ in combination with simple molecules the dependence of vibration energies on junction stretching can provide information on the symmetry of the vibration mode.

Figure 6 shows differential conductance curves that were taken on the same $\mathrm{Pt} / \mathrm{D}_{2}$ junction before (I) and after (II) stretching the junction. Two steps can be seen in each curve, which correspond to two different vibration modes. Due to our limited energy window for inelastic spectroscopy measurements (above $120 \mathrm{mV}$ the $\mathrm{Pt} / \mathrm{H}_{2}$ junction is unstable) we use $\mathrm{D}_{2}$ to shift these vibration energies down toward the center of our measurement window as a result of the twice larger mass. ${ }^{10}$ Focusing on the differences between curves (I) and (II), one observes an increase in the lower energy mode and a decrease in the energy of the higher mode due to junction stretching. This is more clearly seen in the shifts of the peaks/deeps in the conductance derivative $\left(d I^{2} / d^{2} V\right)$.

Figure 7(a) presents sequential vibration-energy shifts due to several stages of stretching. The energy increase in the lower mode (filled circles) can be explained by the response of a transverse mode to stretching, in analogy to a guitar string which gives a higher pitch upon stretching due to an increase in the restoring force. The reduction in the higher mode energy (hollow squares) can be ascribe to the effect of stretching on the longitudinal mode, where the electrodemolecule bond is elongated and weakened resulting in a frequency reduction. This response to stretching agrees closely to the results from density-functional theory calculations. ${ }^{10}$ Note that the latter effect of the weakening of the electrodemolecule bond is also relevant in the case of transverse mode, where following an energy increase due to stretching, further stretching may lead to reduction in the vibration energy. Thus observing an energy reduction by junction stretching is not sufficient for identifying the mode orientation and further tests and comparison with calculations are required.
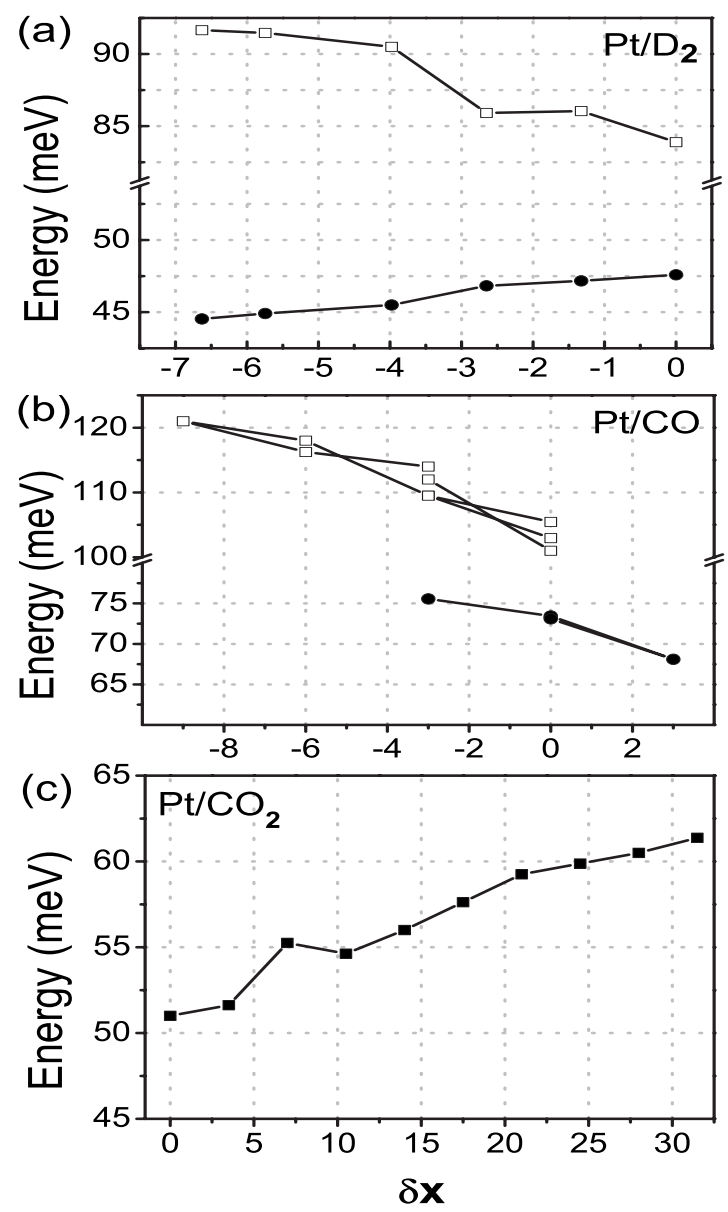

FIG. 7. Vibration energy as a function of the stretching for (a) $\mathrm{Pt} / \mathrm{D}_{2}$, (b) $\mathrm{Pt} / \mathrm{CO}$, and (c) $\mathrm{Pt} / \mathrm{CO}_{2}$ junctions. The stretching distance is measured with respect to the starting point denoted as zero. The unit for the displacement $(\delta x)$ axis is about $0.1 \pm 0.03 \AA$.

In Fig. 7(b) the vibration energies for two modes of a $\mathrm{Pt} / \mathrm{CO}$ junction are reduced by stretching, while the other modes observed in Fig. 5(b) change very little by stretching. We have not succeeded in obtaining a satisfactory interpretation of the results for $\mathrm{Pt} / \mathrm{CO}$ in terms of model calculations. $^{42}$

Figure 7(c) reveals an increase in the energy for the higher vibration mode of a $\mathrm{Pt} / \mathrm{CO}_{2}$ junction. Thus we conclude that this is a transverse mode. No stretching dependence was found in the case of $\mathrm{H}_{2} \mathrm{O}$ and $\mathrm{C}_{6} \mathrm{H}_{6}$ while this property has not been studied for $\mathrm{Pt} / \mathrm{C}_{2} \mathrm{H}_{2}$. Interestingly, up to now stretching dependence was found only in linear molecules.

\section{SUMMERY AND CONCLUDING REMARKS}

Measurements of conductance histograms on molecular junctions for $\mathrm{Pt} / \mathrm{H}_{2}, \mathrm{Pt} / \mathrm{H}_{2} \mathrm{O}, \mathrm{Pt} / \mathrm{CO}, \mathrm{Pt} / \mathrm{CO}_{2}, \mathrm{Pt} / \mathrm{C}_{2} \mathrm{H}_{2}$, and $\mathrm{Pt} / \mathrm{C}_{6} \mathrm{H}_{6}$ show in all cases a main peak around $1 G_{0}$ that indicates the most probable conductance for these junctions. This general behavior is ascribed to the dominant role of the Pt metallic electrode in the bonding to the molecules. Note that this effect is not unique to $\mathrm{Pt}$ electrodes and was ob- 
served in other cases as well. ${ }^{43,44}$ The signature of the molecule is expressed in the fine structure of the histograms such as the exact location of the main peak around $1 G_{0}$ and the peak shape, and in many cases additional peaks are observed. The evolution of the conductance histograms following the introduction of the molecules was found to be similar for the different junctions probably because the process is governed by the metal electrodes.

The molecular nature emerges in the response of the junction to current-induced heating. The junction response can be classified in three scenarios: (i) recovery of a clean Pt junction followed by reconstruction of the molecular junction shortly after the heating $\left(\mathrm{Pt} / \mathrm{H}_{2}\right)$, (ii) recovery of a clean and stable $\mathrm{Pt}$ junction ( $\mathrm{Pt} / \mathrm{CO}, \mathrm{Pt} / \mathrm{CO}_{2}, \mathrm{Pt} / \mathrm{C}_{2} \mathrm{H}_{2}$, and $\mathrm{Pt} / \mathrm{C}_{6} \mathrm{H}_{6}$ ), and (iii) no significant effect $\left(\mathrm{Pt} / \mathrm{H}_{2} \mathrm{O}\right)$. The different behavior is attributed to different molecule diffusion rates at low temperatures and different bonding strength.
Finally, inelastic spectroscopy reveals the characteristic vibration modes of each molecule junction, while the effects of stretching and isotope substitution on the vibration energy allow additional classification of the vibration modes. The distribution of vibration modes given by inelastic spectroscopy is individual for each molecular junction, thus the chemical signature of the suspended molecules is clearly preserved.

\section{ACKNOWLEDGMENTS}

This work is part of the research program of the "Stichting FOM," which is financially supported by NWO. O.T. acknowledges support from IUVSTA-Welch foundation and C.U. acknowledges support through Grant No. MAT200765487 and "Consolider" (Grant No. CSD2007-0010) of the Spanish MEC.
*Present address: Chemical Physics Department, Weizmann Institute of Science, Rehovot 76100, Israel.

${ }^{\dagger}$ Present address: Graduate School of Science, Hokkaido University, Sapporo, Japan, and JST-PRESTO.

*Present address: LT-Nanolab, Departamento de Física Aplicada, Facultad de Ciencias (Fase II), Universidad de Alicante, Campus de S. Vicente del Raspeig, E-03690 Alicante, Spain.

${ }^{1}$ F. Chen, X. Li, J. Hihath, Z. Huang, and N. Tao, J. Am. Chem. Soc. 128, 15874 (2006).

${ }^{2}$ M. Fujihira, M. Suzuki, S. Fujii, and A. Nishikawa, Phys. Chem. Chem. Phys. 8, 3876 (2006).

${ }^{3}$ J. Ulrich, D. Esrail, W. Pontius, L. Venkataraman, D. Millar, and L. H. Doerrer, J. Phys. Chem. B 110, 2462 (2006).

${ }^{4}$ R. H. M. Smit, Y. Noat, C. Untiedt, N. D. Lang, M. C. van Hemert, and J. M. van Ruitenbeek, Nature (London) 419, 906 (2002).

${ }^{5}$ M. Kiguchi, D. Djukic, and J. M. van Ruitenbeek, Nanotechnology 18, 035205 (2007).

${ }^{6}$ O. Tal, M. Krieger, B. Leerink, and J. M. van Ruitenbeek, Phys. Rev. Lett. 100, 196804 (2008).

${ }^{7}$ M. Kiguchi, O. Tal, S. Wohlthat, F. Pauly, M. Krieger, D. Djukic, J. C. Cuevas, and J. M. van Ruitenbeek, Phys. Rev. Lett. 101, 046801 (2008).

${ }^{8}$ N. Agraït, A. L. Yeyati, and J. M. van Ruitenbeek, Phys. Rep. 81, 279 (2003).

${ }^{9}$ W. H. A. Thijssen and M. Strange, J. M. J. aan de Brugh, and J. M. van Ruitenbeek, New J. Phys. 10, 033005 (2008).

${ }^{10}$ D. Djukic, K. S. Thygesen, C. Untiedt, R. H. M. Smit, K. W. Jacobsen, and J. M. van Ruitenbeek, Phys. Rev. B 71, 161402(R) (2005).

${ }^{11}$ H. E. van den Brom and J. M. van Ruitenbeek, Phys. Rev. Lett. 82, 1526 (1999).

${ }^{12}$ D. Djukic and J. M. van Ruitenbeek, Nano Lett. 6, 789 (2006).

${ }^{13}$ B. Ludoph, M. H. Devoret, D. Esteve, C. Urbina, and J. M. van Ruitenbeek, Phys. Rev. Lett. 82, 1530 (1999).

${ }^{14}$ E. Scheer, N. Agraït, J. C. Cuevas, A. L. Yeyati, B. Ludophk, A. Martin-Rodero, G. R. Bollinger, J. M. van Ruitenbeek, and C. Urbina, Nature (London) 394, 154 (1998).
${ }^{15}$ P. Makk, Sz. Csonka, and A. Halbritter, Phys. Rev. B 78, 045414 (2008).

${ }^{16}$ N. Agraït, C. Untiedt, G. Rubio-Bollinger, and S. Vieira, Phys. Rev. Lett. 88, 216803 (2002).

${ }^{17}$ B. C. Stipe, M. A. Rezaei, and W. Ho, Science 280, 1732 (1998).

${ }^{18}$ M. Galperin, M. A. Ratner, and A. Nitzan, J. Chem. Phys. 121, 11965 (2004).

${ }^{19}$ M. Paulsson, T. Frederiksen, and M. Brandbyge, Phys. Rev. B 72, 201101(R) (2005).

${ }^{20}$ J. K. Viljas, J. C. Cuevas, F. Pauly, and M. Häfner, Phys. Rev. B 72, 245415 (2005).

${ }^{21}$ L. de la Vega, A. Martín-Rodero, N. Agraï, and A. Levy Yeyati, Phys. Rev. B 73, 075428 (2006).

${ }^{22}$ A. Halbritter, P. Makk, S. Csonka, and G. Mihaly, Phys. Rev. B 77, 075402 (2008).

${ }^{23}$ Y. Garcia, J. J. Palacios, E. SanFabian, J. A. Verges, A. J. PerezJimenez, and E. Louis, Phys. Rev. B 69, 041402(R) (2004).

${ }^{24}$ C. J. Muller, J. M. van Ruitenbeek, and L. J. de Jongh, Physica C 191, 485 (1992).

${ }^{25}$ C. Untiedt, A. I. Yanson, R. Grande, G. Rubio-Bollinger, N. Agraït, S. Vieira, and J. M. van Ruitenbeek, Phys. Rev. B 66, 085418 (2002).

${ }^{26}$ Milli-Q water: $R>18.2 \mathrm{M} \Omega \mathrm{cm}$ at $25{ }^{\circ} \mathrm{C}$.

${ }^{27} \mathrm{~A}$ different version is composed of two sections: an upper stainless steel tube for an efficient thermal isolation from room temperature and a lower copper tube for homogeneous and fast heating/cooling.

${ }^{28}$ J. W. Quilty, J. Robinson, G. A. Appleby, and A. Edgar, Rev. Sci. Instrum. 78, 083905 (2007).

${ }^{29}$ D. J. Fixsen, P. G. A. Mirel, A. Kogut, and M. Seiffert, Rev. Sci. Instrum. 73, 3659 (2002).

${ }^{30}$ C. Untiedt, M. J. Caturla, M. R. Calvo, J. J. Palacios, R. C. Segers, and J. M. van Ruitenbeek, Phys. Rev. Lett. 98, 206801 (2007).

${ }^{31}$ In control experiments no indication for contaminations were found when the capillary was heated to the relevant temperature without introducing the target molecules.

${ }^{32}$ M. Strange, K. S. Thygesen, and K. W. Jacobsen, Phys. Rev. B 
73, 125424 (2006).

${ }^{33}$ J. Ferrer and V. M. García-Suárez, preceding paper, Phys. Rev. B 80, 085426 (2009).

${ }^{34}$ M. Kiguchi, R. Stadler, I. S. Kristensen, D. Djukic, and J. M. van Ruitenbeek, Phys. Rev. Lett. 98, 146802 (2007).

${ }^{35}$ G. Chiappe, E. Louis, E. V. Anda, and J. A. Verges, Phys. Rev. B 71, 241405(R) (2005).

${ }^{36}$ R. H. M. Smit, C. Untiedt, G. Rubio-Bollinger, R. C. Segers, and J. M. van Ruitenbeek, Phys. Rev. Lett. 91, 076805 (2003).

${ }^{37}$ V. M. Garcia-Suarez, A. R. Rocha, S. W. Bailey, C. J. Lambert, S. Sanvito, and J. Ferrer, Phys. Rev. Lett. 95, 256804 (2005).
${ }^{38}$ S. K. Nielsen, Y. Noat, M. Brandbyge, R. H. M. Smit, K. Hansen, L. Y. Chen, A. I. Yanson, F. Besenbacher, and J. M. van Ruitenbeek, Phys. Rev. B 67, 245411 (2003).

${ }^{39}$ T. N. Todorov, Philos. Mag. B 77, 965 (1998).

${ }^{40}$ D. Segal and A. Nitzan, Chem. Phys. 281, 235 (2002).

${ }^{41}$ S. Alavi, B. Larade, J. Taylor, H. Guo, and T. Seideman, Chem. Phys. 281, 293 (2002).

${ }^{42}$ W. H. A. Thijssen and K. W. Jacobssen (unpublished).

${ }^{43}$ C. Untiedt, D. M. T. Dekker, D. Djukic, and J. M. van Ruitenbeek, Phys. Rev. B 69, 081401(R) (2004).

${ }^{44}$ W. Thijssen, Ph.D. thesis, Universiteit Leiden, 2007. 